\title{
The Timescale of Phenotypic Plasticity and Its Impact on Competition in Fluctuating Environments
}

\author{
Maayke Stomp,$^{1, \star}$ Mark A. van Dijk ${ }^{1, \dagger}$ Harriët M. J. van Overzee, ${ }^{1, \ddagger}$ Meike T. Wortel, ${ }^{1, \S}$ Corrien A. M. Sigon, ${ }^{1, \|}$ \\ Martijn Egas, ${ }^{2, *}$ Hans Hoogveld, ${ }^{3, * *}$ Herman J. Gons, ${ }^{3,+\dagger}$ and Jef Huisman ${ }^{1, \neq \neq}$
}

1. Aquatic Microbiology, Institute for Biodiversity and Ecosystem Dynamics, University of Amsterdam, Nieuwe Achtergracht 127, 1018 WS Amsterdam, The Netherlands;

2. Population Biology, Institute for Biodiversity and Ecosystem Dynamics, University of Amsterdam, P.O. Box 94084, 1090 GB Amsterdam, The Netherlands;

3. Center for Limnology, Netherlands Institute of Ecology (NIOOKNAW), Rijksstraatweg 6, 3631 AC Nieuwersluis, The Netherlands

Submitted January 7, 2008; Accepted May 21, 2008;

Electronically published October 1, 2008

AвSTRACT: Although phenotypic plasticity can be advantageous in fluctuating environments, it may come too late if the environment changes fast. Complementary chromatic adaptation is a colorful form of phenotypic plasticity, where cyanobacteria tune their pigmentation to the prevailing light spectrum. Here, we study the timescale of chromatic adaptation and its impact on competition among phytoplankton species exposed to fluctuating light colors. We parameterized a resource competition model using monoculture experiments with green and red picocyanobacteria and the cyanobacterium Pseudanabaena, which can change its color within $\sim 7$ days by chromatic adaptation. The model predictions were tested in competition experiments, where the incident light color switched between red and

\footnotetext{
* Present address: W. K. Kellogg Biological Station, Michigan State University, Hickory Corners, Michigan 49060; e-mail: m.stomp@uva.nl.

${ }^{\dagger}$ Present address: Center for Limnology, NIOO-KNAW, Rijksstraatweg 6, 3631 AC Nieuwersluis, The Netherlands; e-mail: m.vandijk@nioo.knaw.nl.

₹ Present address: Wageningen Institute for Marine Resources and Ecosystem Studies, P.O. Box 68, 1970 AB IJmuiden, The Netherlands; e-mail: harriet.vanoverzee@wur.nl.

`E-mail: meike.wortel@student.uva.nl.

|| E-mail: c.a.m.sigon@uva.nl.

\#E-mail: c.j.m.egas@uva.nl.

** E-mail: h.hoogveld@nioo.knaw.nl.

${ }^{\dagger \dagger}$ Deceased.

\$ Corresponding author; e-mail: j.huisman@uva.nl.
}

Am. Nat. 2008. Vol. 172, pp. E169-E185. (c) 2008 by The University of Chicago. 0003-0147/2008/17205-50153\$15.00. All rights reserved. DOI: $10.1086 / 591680$ green at different frequencies (slow, intermediate, and fast). Pseudanabaena (the flexible phenotype) competitively excluded the green and red picocyanobacteria in all competition experiments. Strikingly, the rate of competitive exclusion was much faster when the flexible phenotype had sufficient time to fully adjust its pigmentation. Thus, the flexible phenotype benefited from its phenotypic plasticity if fluctuations in light color were relatively slow, corresponding to slow mixing processes or infrequent storms in their natural habitat. This shows that the timescale of phenotypic plasticity plays a key role during species interactions in fluctuating environments.

Keywords: adaptive dynamics, cyanobacteria, phycocyanin, phycoerythrin, resource competition theory, Synechococcus.

Fluctuations in environmental conditions pose serious challenges to organisms. Many organisms respond to environmental changes by physiological and morphological adaptations. This flexible strategy, known as phenotypic plasticity, may improve their fitness in the new environments (Agrawal 2001). For example, plants increase their leaf area during periods of reduced light (Sultan and Bazzaz 1993), cladocerans develop armored helmets in the presence of predators (Woltereck 1909; Laforsch and Tollrian 2004), and some green algae aggregate into colonies to reduce their edibility for grazers (Hessen and Van Donk 1993; Lampert et al. 1994).

Intuitively, phenotypic plasticity seems a suitable strategy to cope with environmental fluctuations. However, adaptation takes time. If adaptation is too slow, organisms will not be able to keep up with changes in their environment, resulting in a permanent mismatch between the physiology of the organisms and their environmental conditions. Indeed, theory shows that adaptation can even be disadvantageous when it has a strong time delay (Padilla and Adolph 1996; Gabriel 2005). Yet, although many studies have investigated phenotypic plasticity in fluctuating environments (e.g., Chesson et al. 2004; Egas et al. 2004; Abrams 2006a, 2006b; Gélinas et al. 2007; Van der Stap et al. 2007), the timescale of phenotypic adaptation has received surprisingly little attention (Miner et al. 2005).

The colorful process of complementary chromatic ad- 
aptation is a spectacular form of phenotypic plasticity found in many cyanobacteria. During chromatic adaptation, cyanobacteria change their pigment composition and thereby their color (Gaiducov 1902; Tandeau de Marsac 1977; Grossman et al. 1993; Kehoe and Gutu 2006). In the presence of green light, they turn their accessory pigments into the complementary color. That is, they become red. Conversely, in the presence of red light, they turn green. This complementary adaptation optimizes light absorption and thus favors their photosynthesis and growth. The ecological significance of the spectral tuning of the pigment composition to the ambient light color is debated for macroalgae (Dring 1981; Ramus 1983). However, theory, competition experiments, and field studies have shown that the underwater light color is a major selective factor for phototrophic microorganisms (e.g., Montesinos et al. 1983; Béjà et al. 2001; Rocap et al. 2003; Stomp et al. 2004). For instance, we showed in a series of competition experiments that red picocyanobacteria win the competition in green light, green picocyanobacteria win the competition in red light, and red and green strains can coexist in the full light spectrum provided by white light (Stomp et al. 2004). This matches their field distributions: red cyanobacteria dominate in clear waters in which green light penetrates the deepest, whereas green cyanobacteria dominate in turbid waters in which red light penetrates the deepest (Pick 1991; Vörös et al. 1998; Wood et al. 1998; Vila and Abella 2001; Stomp et al. 2007a, 2007b). Coexistence of red and green cyanobacteria is widespread in waters of intermediate turbidity (Stomp et al. 2007b).

Here, we study the timescale of phenotypic plasticity in a fluctuating environment and its impact on interspecific competition. We use three cyanobacteria isolated from the Baltic Sea. One of these species is capable of complementary chromatic adaptation and can change its color from red to green (and vice versa) within a week. The other two species are a green and a red picocyanobacterium, both with a fixed pigment composition. We first develop a resource competition model to predict how these species would interact in a fluctuating environment where the light color switches between red and green. Subsequently, we perform monoculture experiments to estimate the growth parameters and light absorption characteristics of the three species. Finally, the model predictions are tested in a series of competition experiments in which the light color fluctuates at low, intermediate, and high frequencies. The results show how the interplay between the timescale of phenotypic plasticity and the timescale of environmental fluctuations affects the rate of competitive exclusion.

\section{Competition Model}

Our theoretical framework on competition for light originates from the competition model developed by Huisman and Weissing $(1994,1995)$. Their model assumes a direct coupling between changes in phytoplankton population densities and changes in light availability caused by phytoplankton shading. The model treats light as a single resource and predicts that, in well-mixed waters, the species with the lowest critical light intensity will be the superior competitor for light. The critical light intensity is analogous to the $\mathrm{R}^{*}$ concept (Tilman 1982) in competition for nutrients. It can be measured in monoculture experiments as the light intensity penetrating through the monoculture at steady state. The model has been successfully applied to species with similar light absorption spectra (Huisman et al. 1999a; Passarge et al. 2006; Agawin et al. 2007). Recently, the competition model of Huisman and Weissing was extended to cover the full light spectrum (Stomp et al. 2004, 2007a, 2007b). That is, light is treated as a spectrum of resources. This spectral model predicts that species can coexist if they develop different pigments to utilize different parts of the light spectrum (Stomp et al. 2004) and will provide the basis for the current study.

We consider a well-mixed water column in which the phytoplankton species are homogeneously distributed. Nutrients are supplied in excess, so that the species compete for light only. The depth is indicated by $z$, scaled from 0 at the surface to a maximum depth $z_{\mathrm{m}}$ at the bottom of the water column. The water column is illuminated from above, with an incident light spectrum $I_{\text {in }}(\lambda)$, where $\lambda$ is the wavelength. The light spectrum changes with depth as a result of selective absorption of different parts of the underwater light spectrum by the phytoplankton community and by the background turbidity caused by water and other nonphytoplankton components. Let $I(\lambda, z)$ denote the light spectrum at depth $z$. According to LambertBeer's law, the change of the underwater light spectrum can be described as

$$
I(\lambda, z)=I_{\text {in }}(\lambda) \exp \left(-\sum_{i=1}^{n} k_{i}(\lambda) N_{i} z-K_{\mathrm{bg}}(\lambda) z\right)
$$

(Sathyendranath and Platt 1989; Kirk 1994; Stomp et al. 2004), where $k_{i}(\lambda)$ is the specific light absorption spectrum of phytoplankton species $i, N_{i}$ is the population density of phytoplankton species $i, n$ is the total number of phytoplankton species, and $K_{\mathrm{bg}}(\lambda)$ is the background light absorption spectrum. Examples of light absorption spectra of phytoplankton species are illustrated in figure 1. The two peaks at $430 \mathrm{~nm}$ (blue light) and $680 \mathrm{~nm}$ (red light), shared by all three species, correspond to light absorption by the green pigment chlorophyll $a$. The peak at $560-570$ $\mathrm{nm}$ corresponds to the red pigment phycoerythrin, which effectively absorbs green light. The peak at $620-630 \mathrm{~nm}$ corresponds to the pigment phycocyanin, which absorbs 

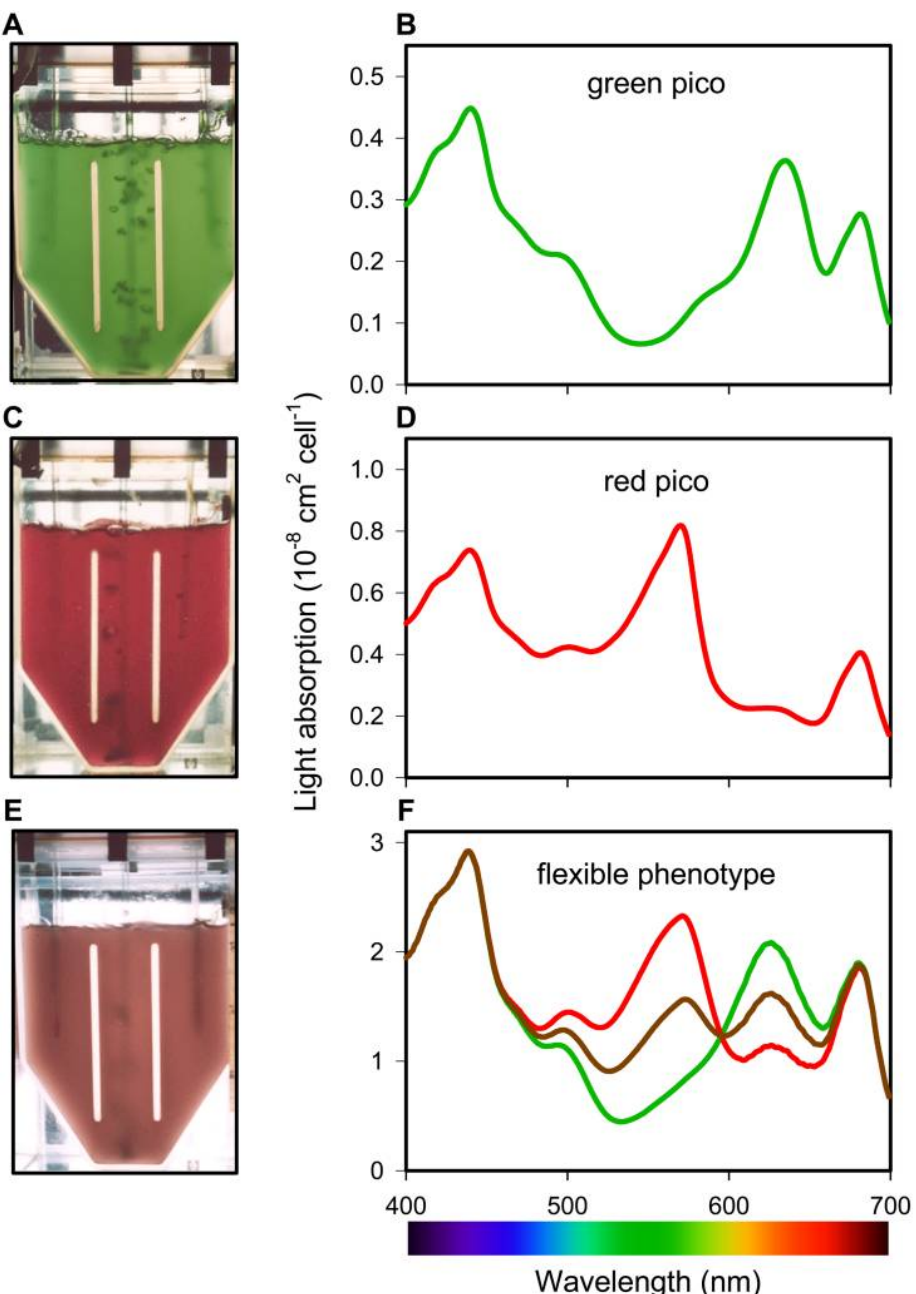

Figure 1: Optical characteristics of Synechococcus CCY9201 (A, B; green pico), Synechococcus strain CCY9202 (C, D; red pico), and Pseudanabaena CCY9509 ( $E, F$; flexible phenotype). Panels on the left show monocultures of the three strains in light-limited chemostats. Panels on the right show their light absorption spectra. The flexible phenotype can tune its light absorption spectrum to the prevailing light conditions by changing its cellular content of phycoerythrin (absorption peak at $\sim 560 \mathrm{~nm}$ ) and phycocyanin (absorption peak at $\sim 620 \mathrm{~nm}$ ). In $F$, the red line indicates the light absorption spectrum of the flexible phenotype when fully adapted to green light, the green line indicates its light absorption spectrum when fully adapted to red light, and the brown line indicates its light absorption spectrum measured midway during complementary chromatic adaptation.

orange-red light. Note from figure 1 that the color that is least absorbed by a phytoplankton species remains available for other species.

The number of photons absorbed by a single cell of species $i$ (over the photosynthetically active radiation [PAR] range; 400-700 $\mathrm{nm}$ ) at depth $z, \gamma_{i}(z)$, depends on the match between the underwater light spectrum and its light absorption spectrum and can be calculated as

$$
\gamma_{i}(z)=\int_{400}^{700} I(\lambda, z) k_{i}(\lambda) d \lambda
$$

(Stomp et al. 2004, 2007b). We assume that the specific growth rate of a phytoplankton species is an increasing, saturating function of the number of photons it has absorbed. The specific growth rate thus calculated varies with depth and is integrated over the entire water column to obtain the total population growth rate (Huisman and Weissing 1994). Accordingly, the population dynamics of species $i$ can be described as

$$
\frac{d N_{i}}{d t}=\left[\frac{1}{z_{\mathrm{m}}} \int_{0}^{z_{\mathrm{m}}} \frac{p_{\max , i} \gamma_{i}(z)}{\left(p_{\max , i} / \varphi_{i}\right)+\gamma_{i}(z)} d z\right] N_{i}-L_{i} N_{i}
$$


(Sathyendranath and Platt 1989; Stomp et al. 2007b), where $\varphi_{i}$ is the photosynthetic efficiency (quantum yield) of species $i, p_{\max , i}$ is its maximum specific growth rate, and $L_{i}$ is its specific loss rate due to factors such as grazing and sinking.

In terms of competition theory, the light spectrum can be interpreted as a spectrum of resources, analogous to the spectrum of seed sizes available for Darwin's finches (Stomp et al. 2007a, 2007b). The light absorption spectra of species define which part of the light spectrum they can utilize. Species that are not capable of chromatic adaptation have a fixed light absorption spectrum, whereas species capable of complementary chromatic adaptation have a flexible light absorption spectrum due to variations in phycocyanin and phycoerythrin content (fig. $1 F$ ). There are different types of complementary chromatic adaptation (Grossman et al. 1993; Kehoe and Gutu 2006). In our case, we focus on Type III, in which the total pool of phycoerythrin (PE) and phycocyanin (PC) remains constant, but the ratio of these two pigments is adjusted to the prevailing underwater light spectrum. Accordingly, we describe the specific light absorption spectrum of the flexible species as

$$
k_{i}(\lambda)=x_{i} k_{\mathrm{PE}}(\lambda)+\left(1-x_{i}\right) k_{\mathrm{PC}}(\lambda)+k_{\text {other }}(\lambda)
$$

where $x_{i}$ is the fraction phycoerythrin (i.e., $\mathrm{PE} /(\mathrm{PE}+$ $\mathrm{PC})), k_{\mathrm{PE}}(\lambda)$ is the absorption spectrum of a cell with phycoerythrin only, $k_{\mathrm{PC}}(\lambda)$ is the absorption spectrum of a cell with phycocyanin only, and $k_{\text {other }}(\lambda)$ is the absorption spectrum due to other pigments in the cell (i.e., chlorophyll $a$, carotenoids).

We assume that the flexible species changes its phenotype to maximize its specific growth rate under the prevailing environmental conditions (Metz et al. 1992; Abrams 1999; Egas et al. 2005). In our case, this implies that the species will adjust its phycocyanin and phycoerythrin content to maximize the number of photons it absorbs (Stomp et al. 2004):

$$
\frac{d x_{i}}{d t}=\alpha_{i} \frac{\varphi_{i}}{z_{\mathrm{m}}} \int_{0}^{z_{\mathrm{m}}} \frac{\partial \gamma_{i}\left(z, x_{i}\right)}{\partial x_{i}} d z
$$

where $\alpha_{i}$ measures the rate of chromatic adaptation. Note from this equation that the phycoerythrin fraction $\left(x_{i}\right)$ will change in the direction that yields more light absorption (i.e., a higher $\gamma_{i}$ ). The parameter $\alpha_{i}$ is of central importance in this study because it determines the timescale of chromatic adaptation. Numerical simulations of the model were based on a fourth-order Runge-Kutta procedure for time integration and Simpson's rule for depth integration.

\section{Methods}

Species

The species chosen for the experiments were the green picocyanobacterium Synechococcus strain CCY9201 (formerly known as Synechococcus BS4), the red picocyanobacterium Synechococcus strain CCY9202 (formerly known as Synechococcus BS5), and the filamentous cyanobacterium Pseudanabaena strain CCY9509. The last species can change its color by complementary chromatic adaptation (Type III; Grossman et al. 1993; Kehoe and Gutu 2006). In this article, the three species are referred to as the green pico, red pico, and flexible phenotype, respectively. All three species were isolated from the Baltic Sea (Ernst et al. 2003; Haverkamp et al. 2008; Acinas et al. 2008). The red and green Synechococcus strains were also used in earlier competition experiments (Stomp et al. 2004).

All three species contain the green pigment chlorophyll $a$, with absorption peaks at $430 \mathrm{~nm}$ and $680 \mathrm{~nm}$. In addition, the green pico (fig. 1A) contains high concentrations of the pigment phycocyanin (PC), which absorbs orange-red light at 620-630 $\mathrm{nm}$ (fig. $1 B$ ). The red pico (fig. 1C) contains a little phycocyanin, but its main accessory pigment is phycoerythrin (PE), which absorbs green light at 560-570 nm (fig. $1 D$ ). The flexible phenotype (fig. $1 E$ ) can adjust its concentration of PE and PC to the color of the prevailing light spectrum (fig. 1F; Tandeau de Marsac 1977; Grossman et al. 1993; Kehoe and Gutu 2006) and thereby changes its own color from red to green and vice versa.

\section{Experimental Setup}

Experiments were performed in chemostats specifically designed to study light-limited growth (Huisman et al. 1999a; Stomp et al. 2004; Passarge et al. 2006). The dilution rate of the chemostats was set at $D=0.014 \mathrm{~h}^{-1}$. Flat chemostat vessels were illuminated from the side. Hence, the mixing depth of the chemostats was defined by the width of the vessels, which equaled $z_{\mathrm{m}}=5 \mathrm{~cm}$. The light source consisted of fluorescent tubes with a white light spectrum (Philips TLD 18W/965). Green light conditions were obtained by a dark green filter (Lee filter 124, Andover, UK) placed between the light source and the chemostat vessel, while red light conditions were obtained by a red filter (Lee filter 26). Fluctuations in incident light color were generated by time clock-controlled switching between two light sources placed on opposite sides of the chemostats, where one light source used a red filter and the other light source used a green filter (fig. 2). Monoculture experiments of the three species were carried out under continuous green light and under continuous red light. Competition experiments, in which all three species were mixed 
A

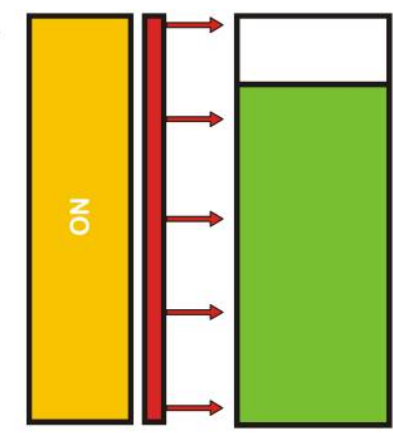

B

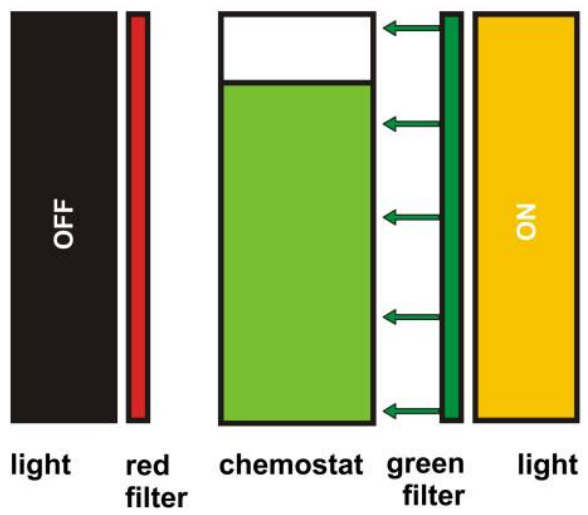

Figure 2: Schematic view of the experimental setup. $A$, Chemostat under red light conditions. $B$, Chemostat under green light conditions. Fluctuations of incident light color were obtained by time clock-controlled switching between the left and right light sources.

together, were carried out under continuous green light, continuous red light, and fluctuating red/green light conditions. The fluctuations in light color were characterized by their periodicity $(T)$. We applied the following three fluctuation regimes: $T=0.5$ days $(6 \mathrm{~h} \mathrm{red} / 6 \mathrm{~h}$ green $)$, $T=4$ days $(2 \mathrm{~d}$ red/2 $\mathrm{d}$ green $)$, and $T=28$ days $(14 \mathrm{~d}$ $\mathrm{red} / 14 \mathrm{~d}$ green).

The incident light intensity $\left(I_{\text {inPAR }}\right)$ and the light intensity penetrating through the cultures $\left(I_{\text {outPAR }}\right)$ were measured with a Licor LI-190SA quantum sensor (Lincoln, NE), which integrates the measured number of photons over the entire PAR range $(400-700 \mathrm{~nm})$. In all experiments, we supplied an incident light intensity of $I_{\text {inPAR }}=40$ $\mu \mathrm{mol} \mathrm{m}{ }^{-2} \mathrm{~s}^{-1}$ to the chemostat vessel. The spectra of the incident light, $I_{\text {in }}(\lambda)$, and the light penetration through the vessel, $I_{\text {out }}(\lambda)$, were measured by a Licor LI-1800 spectroradiometer. Light absorption spectra of the species were measured by an Aminco DW-2000 double-beam spectrophotometer (SLM Instruments, Urbana, IL), using mineral medium without phytoplankton as a control.

\section{Population Densities}

Population densities of the species were counted by flow cytometry (Jonker et al. 1995; Stomp et al. 2004). Because Pseudanabaena was much larger than the two picocyanobacteria, direct comparison of the population densities does not provide a good representation of the relative abundances of the different species in the species mixtures. To quantify the contribution of each species in the context of competition for light, we therefore expressed the population densities of the species in terms of their total light absorption. For calculation of the total light absorption by each species and further details on the experimental conditions and analysis, see the appendix.

\section{Parameter Estimation}

Model parameters were estimated from the experiments. The preceding paragraphs already specified our measurements of dilution rate $(D)$, mixing depth $\left(z_{\mathrm{m}}\right)$, incident light spectrum $\left(I_{\text {in }}(\lambda)\right)$, and the light absorption spectra of the species $\left(k_{i}(\lambda)\right)$. Calculation of the background turbidity $\left(K_{\mathrm{bg}}(\lambda)\right)$ is described in the appendix. We assumed that specific loss rates of the species were dominated by the dilution rate (i.e., $L_{i}=D$ for all species).

The remaining model parameters were the photosynthetic efficiencies $(\varphi)$ and the maximum specific growth rates $\left(p_{\max }\right)$ of all three species and the rate of chromatic adaptation of the flexible phenotype $(\alpha)$. We estimated the model parameters $\varphi$ and $p_{\max }$ by calibration of the model predictions to time series of population density and light penetration measured in monoculture experiments. The model calibration was simultaneously applied to monoculture experiments under continuous red light and monoculture experiments under continuous green light to obtain parameter estimates for $\varphi$ and $p_{\max }$ that applied to both red light and green light conditions. For this purpose, the data of population density and light penetration were first $\log$ transformed to homogenize the variances and subsequently normalized using the total sum of squares as a weighting factor to give equal weight to each of these variables in the calibration procedure. Parameter estimates were obtained by fitting the model predictions to these log-transformed normalized data by minimization of the residual sum of squares, using the Gauss-MarquardtLevenberg algorithm. The model calibration was performed with the software package PEST (Watermark Numerical Computing, Brisbane).

Subsequently, the rate of chromatic adaptation of the flexible phenotype $(\alpha)$ was estimated by fitting the model to two separate monoculture experiments. In one experiment, a culture adjusted to green light was exposed to red light for 1 week, while in the other experiment, a 
culture adjusted to red light was exposed to green light for 1 week. From these experiments, the parameter $\alpha$ was estimated using the same calibration technique as described above. The species parameters that were estimated from the monoculture experiments were used to predict the population dynamics in the competition experiments.

\section{Rate of Competitive Exclusion}

To examine the dynamics in each competition experiment, we calculated the rate of competitive exclusion (RCE). This rate was defined as

$$
\mathrm{RCE}=\frac{\Delta \ln (\text { winner/loser })}{\Delta t}
$$

(Grover 1988; Passarge et al. 2006), where winner and loser are the population densities of the winning and losing species during the course of competition, measured from the onset of the decline of the loser. Accordingly, RCE can be estimated from the measured population densities by linear regression of $\ln$ (winner/loser) versus time.

\section{Results}

\section{Monoculture Experiments}

In monoculture, the green pico, red pico, and flexible phenotype were all able to grow under both continuous red and continuous green light (fig. 3). After inoculation, the population densities increased, absorbed more light, and thus decreased the light intensity penetrating through the vessel. This continued until steady state was reached. The population of the green pico reached steady state within $\sim 8$ days when grown in red light, while it took $\sim 21$ days to reach steady state in green light (fig. $3 A, 3 B$ ). The steady state population density of the green pico and its total light absorption were higher in red light than in green light (table 1). We define the spectrally integrated light intensity penetrating through a steady state monoculture as the critical light intensity $\left(I_{\text {outPAR }}{ }^{*}\right.$; sensu Huisman and Weissing 1994). The critical light intensity of the green pico was much lower in red light than in green light (fig. $3 A, 3 B$; table 1 ) as a result of its higher population density in red light and its more efficient absorption of red light (fig. 1B).

The population of the red pico reached steady state within $\sim 8$ days when grown in green light, while it took $\sim 12$ days in red light (fig. $3 C, 3 D$ ). The steady state population density of the red pico was similar in green light and in red light (table 1). The total light absorption of the red pico was higher in green light than in red light (table 1) because of its more efficient absorption of green light (fig. 1D). As a result, the critical light intensity of the red pico was much lower in green light than in red light (fig. $3 C$, $3 D$; table 1 )

The population of the flexible phenotype reached steady state within $\sim 10$ days in both red light and green light (fig. $3 E, 3 F)$. In red light, the flexible phenotype produced mainly phycocyanin pigments and thereby turned its color into green (fig. $1 F)$. Conversely, in green light, the flexible phenotype produced mainly phycoerythrin pigments, turning its color into red. The steady state population density of the flexible phenotype, and hence its total light absorption, was quite similar in both red light and green light (table 1). The critical light intensity of the flexible phenotype was lower in red light than in green light (fig. $3 E$, $3 F$; table 1). Under green light, the flexible phenotype had a critical light intensity similar to that of the red pico but lower than that of the green pico. Conversely, under red light, the flexible phenotype had a critical light intensity similar to that of the green pico but lower than that of the red pico.

The data from these monoculture experiments were used to calibrate the model parameters of the species (table 2). For this purpose, the model was fitted simultaneously to the time courses of the population densities and light penetration under both red and green light conditions. The calibrated model fitted quite well with the experimental data (fig. 3).

To estimate the rate of chromatic adaptation of the flexible phenotype, we simply exchanged the colored light filters of the two monoculture experiments (fig. $3 E, 3 F$ ). When the green light-adapted culture was transferred to red light, it gradually replaced its phycoerythrin by phycocyanin and thereby turned its color from red to green in $\sim 7$ days (fig. $4 A$ ). Conversely, when the red lightadapted culture was transferred to green light, it gradually replaced its phycocyanin by phycoerythrin and thereby turned its color from green to red in 8-9 days (fig. 4B). Thus, the rate of chromatic adaptation was rather similar in both directions, although possibly slightly faster from red to green pigmentation than from green to red pigmentation (table 2).

\section{Competition Experiments}

We performed competition experiments with mixtures of the red pico, green pico, and flexible phenotype under continuous red light, continuous green light, and fast, intermediate, and slow fluctuations in light color. Under continuous red light, the red pico was rapidly excluded, while the green pico and flexible phenotype coexisted (fig. $5 A$ ). This is consistent with the critical light intensities for red light measured in monoculture, which were much lower for the flexible phenotype and green pico than for 


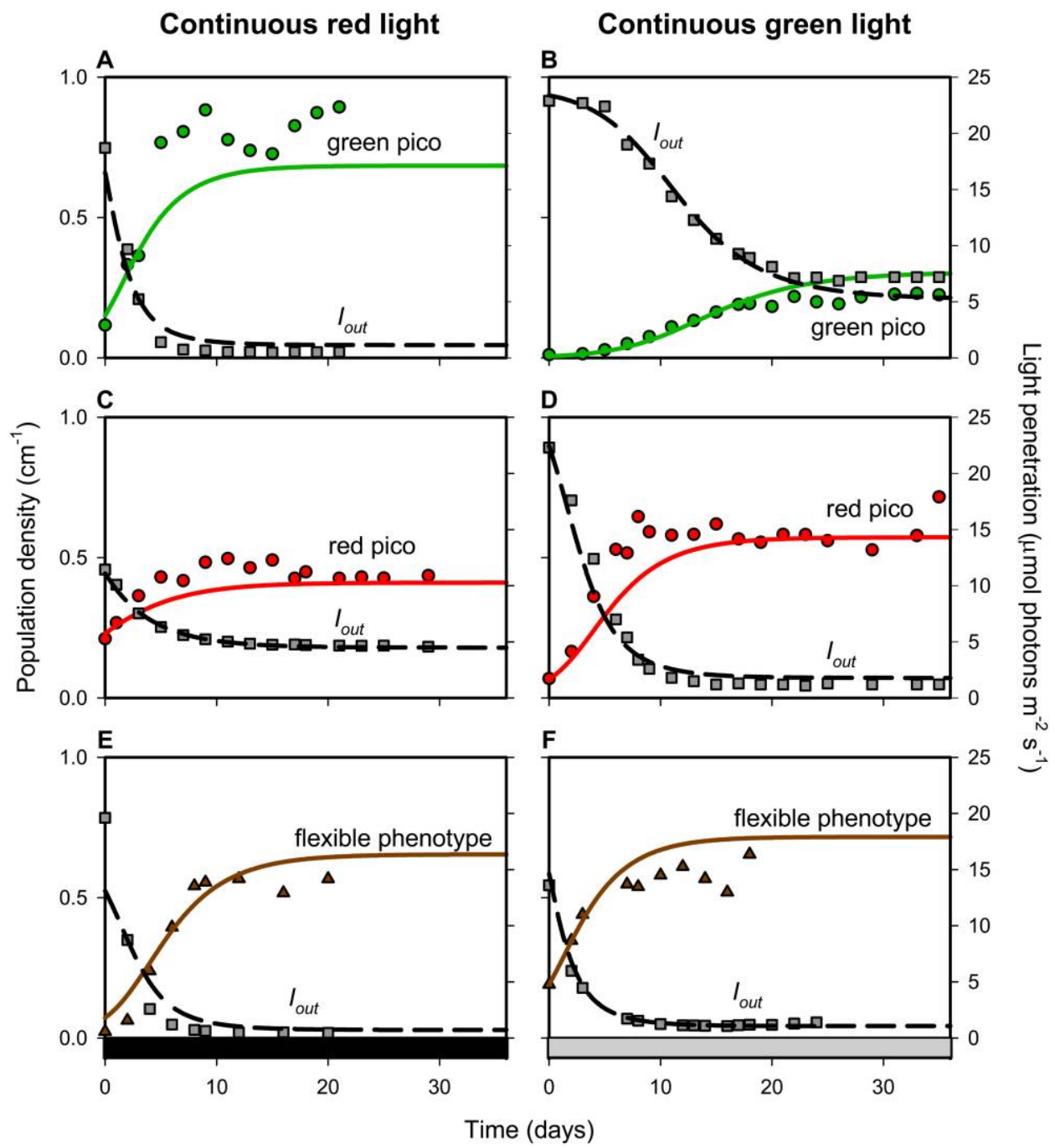

Figure 3: Monoculture experiments. A, B, Green pico (green circles). $C, D$, Red pico (red circles). E, F, Flexible phenotype (brown triangles). Panels on the left show monocultures grown in continuous red light, while panels on the right show monocultures grown in continuous green light. Gray squares indicate the light intensity penetrating through the cultures. Solid lines indicate the population densities predicted by the model. Dashed lines indicate the predicted light penetration. Bars at the bottom of the panels indicate illumination by red light (black bar) and green light (gray bar). Population densities of the species are expressed by their light absorption. For parameter values, see table 2 .

the red pico (table 1). The critical light intensities for red light of the flexible phenotype and green pico were very similar, and their coexistence is probably an example of neutral coexistence. Conversely, under continuous green light, the flexible phenotype rapidly excluded the green pico, while the red pico was displaced more slowly (fig. $5 B$ ). This is consistent with the critical light intensities for green light, which were much lower for the flexible phenotype and red pico than for the green pico (table 1). Although the critical light intensities for green light of the flexible phenotype and red pico were rather similar, the competition experiment did not reveal neutral coexistence of these two species but showed that the flexible phenotype was a slightly stronger competitor for green light than the red pico.

In all competition experiments under fluctuating light conditions, the flexible phenotype excluded both the green and red picos (fig. 6). When exposed to fast fluctuations in light color ( $T=0.5$ days), the flexible phenotype gradually developed a pigment composition rich in phycoerythrin; fluctuations in pigment composition were not detectable in this experiment (fig. $6 G$ ). Under intermediate fluctuations in light color ( $T=4$ days), the flexible phenotype changed its pigment composition but had insuf- 
Table 1: Steady state traits of the phytoplankton species measured in monoculture experiments (means $\pm \mathrm{SD})$

\begin{tabular}{lrrr}
\hline & Green pico & Red pico & Flexible phenotype \\
\hline Population density $\left(\times 10^{8}\right.$ counts $\left.\mathrm{mL}^{-1}\right):$ & & & \\
$\quad$ Red light & $3.59 \pm .36$ & $1.34 \pm .09$ & $.45 \pm .02$ \\
$\quad$ Green light & $1.66 \pm .12$ & $1.31 \pm .13$ & $.45 \pm .04$ \\
Light absorption $\left(\mathrm{cm}^{-1}\right):$ & $.82 \pm .07$ & $.45 \pm .03$ & $.55 \pm .02$ \\
$\quad$ Red light & $.22 \pm .01$ & $.60 \pm .05$ & $.59 \pm .05$ \\
$\quad$ Green light & $.50 \pm .02$ & $4.69 \pm .07$ & $.51 \pm .07$ \\
Critical light intensity $\left(\mu \mathrm{mol} \mathrm{m}^{-2} \mathrm{~s}^{-1}\right):$ & $7.09 \pm .15$ & $1.21 \pm .06$ & $1.18 \pm .10$ \\
$\quad$ Red light & &
\end{tabular}

ficient time to fully adjust its pigmentation to the prevailing light color. Hence, the pigment composition of the flexible phenotype fluctuated with relatively small amplitude (fig. 6H). Under slow fluctuations in light color $(T=28$ days), there was sufficient time for the flexible phenotype to fully adjust its pigmentation before the light color was changed again. In this experiment, the population density, light penetration, and pigment composition of the flexible phenotype fluctuated strongly (fig. $6 C, 6 F$, $6 I)$.

The model predictions, based on the parameters estimated from the monoculture experiments, were in good agreement with the competition experiments (fig. 6). However, especially in the fast and intermediate fluctuation regimes, the flexible phenotype had a slightly higher phycoerythrin fraction than predicted by the model (fig. $6 G, 6 H)$. Furthermore, in the slow fluctuation regime, the rate at which the flexible phenotype adjusted its phycoerythrin fraction to green light was consistent with the model predictions, while the adjustment to red light was somewhat more slowly than predicted (fig. 6I).

\section{At What Timescale Is Chromatic Adaptation Advantageous?}

Summarizing the experimental results, we found that the amplitude of the variation in pigment composition of the flexible phenotype increased with longer periods of environmental fluctuation (fig. 7A). The flexible phenotype required $\sim 7$ days to turn its color from red to green, while it required 8-9 days to turn its color from green to red (fig. 4). Accordingly, the model predicts that at light fluctuations with a period exceeding $\sim 14$ days (i.e., 7 days red and 7 days green), the flexible phenotype could fully adjust its pigmentation to red light conditions before the light color switched to green again (fig. 7A). Slightly longer periods of fluctuation ( $T>18$ days; i.e., 9 days red and 9 days green) were required for the flexible phenotype to fully adjust to green light conditions (fig $7 A$ ). Note that, at fast fluctuations ( $T=0.5$ days), the flexible phenotype had more phycoerythrin than predicted by the model (fig. $7 A)$.

Intuitively, one might think that larger amplitudes in pigmentation of the flexible phenotype would enhance its tuning to red and green light conditions and thus would promote its light absorption. However, the model predicts that for $T<14$ days (i.e., the range of periodicities where the amplitude in pigmentation increased with $T$; see fig. $7 A$ ), the time-averaged light absorption by the flexible phenotype remains constant (fig. $7 B$ ). The explanation is that, with increasing $T$, the flexible phenotype makes more phycoerythrin to absorb more green light, but as a consequence, it will also be more poorly adapted once the red light is switched on again. Thus, the gain in light absorption by a better tuning to green light is offset by a reduced light absorption when the light color is switched back to red light. As a result, the time-averaged light absorption by the flexible phenotype remains constant over $T$ (for $T<14$ days). At longer periods ( $T>14$ days), there is sufficient time for the flexible phenotype to fully adjust its pigmentation to the prevailing light color, and the flexible phenotype starts to benefit from chromatic adaptation (fig. $7 B$ ). More specifically, the model predicts that, for $T>14$ days, the flexible phenotype will spend relatively less time changing its pigmentation while it can benefit for longer time spans from its optimal tuning to the prevailing light color. Hence, for $T>14$ days, the timeaveraged light absorption increases with $T$ until it approaches an asymptote at which the time spent on chromatic adaptation is negligible (fig. $7 \mathrm{~B}$ ). These model predictions were confirmed by the experiments. Although the standard deviations are high, the data show that light absorption by the flexible phenotype was higher at long periods of fluctuation $(T=28$ days $)$ than at shorter periods $(T=0.5$ and 4 days). Also in line with the model predictions, light absorption by the flexible phenotype was higher during red light conditions than during green light conditions.

The rate at which the flexible phenotype competitively excludes the green and red picos is tightly coupled to its efficiency in absorbing red and green light. Since the green 
Table 2: Parameter values and their interpretations

\begin{tabular}{|c|c|c|c|}
\hline Symbol & Interpretation & Unit & Value \\
\hline \multicolumn{4}{|l|}{ Independent variables: } \\
\hline$t$ & Time & $\mathrm{h}$ & $\cdots$ \\
\hline$z$ & Depth & $\mathrm{cm}$ & $\ldots$ \\
\hline$\lambda$ & Wavelength & $\mathrm{nm}$ & $\ldots$ \\
\hline \multicolumn{4}{|l|}{ Dependent variables: } \\
\hline$N_{i}$ & Population density of species $i$ & cells $\mathrm{cm}^{-3}$ & $\ldots$ \\
\hline$A_{i}$ & Light absorption by species $i$ & $\mathrm{~cm}^{-1}$ & $\ldots$ \\
\hline$\gamma_{i}(z)$ & Absorbed photons by species $i$ & $\mu \mathrm{mol} \mathrm{s}^{-1}$ cell $^{-1}$ & $\ldots$ \\
\hline$I(\lambda, z)$ & Underwater light spectrum & $\mu \mathrm{mol} \mathrm{m}{ }^{-2} \mathrm{~s}^{-1} \mathrm{~nm}^{-1}$ & $\ldots$ \\
\hline$x_{i}$ & Fraction phycoerythrin of species $i$ & Dimensionless & $\ldots$ \\
\hline \multicolumn{4}{|l|}{ Parameters: } \\
\hline$I_{\text {in }}(\lambda)$ & Spectrum of incident light & $\mu \mathrm{mol} \mathrm{m}{ }^{-2} \mathrm{~s}^{-1} \mathrm{~nm}^{-1}$ & a \\
\hline$I_{\text {inPAR }}$ & PAR-integrated incident light intensity & $\mu \mathrm{mol} \mathrm{m} \mathrm{m}^{-2} \mathrm{~s}^{-1}$ & $40^{\mathrm{a}}$ \\
\hline$I_{\text {outPAR }}$ & PAR-integrated penetrating light intensity & $\mu \mathrm{mol} \mathrm{m} \mathrm{m}^{-2} \mathrm{~s}^{-1}$ & a \\
\hline$K_{\mathrm{bg}}(\lambda)$ & Absorption spectrum of background turbidity & $\mathrm{cm}^{-1}$ & a \\
\hline$k_{i}(\lambda)$ & Absorption spectrum of species $i$ & $\mathrm{~cm}^{2}$ cell $^{-1}$ & Figure $1^{\mathrm{a}}$ \\
\hline$k_{\mathrm{PE}}(\lambda)$ & Absorption spectrum of phycoerythrin & $\mathrm{cm}^{2}$ cell $^{-1}$ & $\mathrm{~b}$ \\
\hline$k_{\mathrm{PC}}(\lambda)$ & Absorption spectrum of phycocyanin & $\mathrm{cm}^{2}$ cell $^{-1}$ & b \\
\hline$k_{\text {other }}(\lambda)$ & Absorption spectrum of chlorophyll and carotenoid pigments & $\mathrm{cm}^{2}$ cell $^{-1}$ & $\mathrm{~b}$ \\
\hline$z_{\mathrm{m}}$ & Depth of the water column & $\mathrm{cm}$ & $5.0^{\mathrm{a}}$ \\
\hline$L_{i}$ & Specific loss rate of species $i$ & $\mathrm{~h}^{-1}$ & $.014^{\mathrm{a}}$ \\
\hline$p_{\max , i}$ & Maximum specific growth rate of species $i$ & $\mathrm{~h}^{-1}$ & $.080^{\mathrm{c}}$ \\
\hline$\varphi_{i}$ & Photosynthetic efficiency & cells $(\mu \mathrm{mol})^{-1}$ & \\
\hline Green pico & & & $2.4 \times 10^{6 \mathrm{~b}}$ \\
\hline Red pico & & & $1.0 \times 10^{6 \mathrm{~b}}$ \\
\hline Flexible phenotype & & & $4.2 \times 10^{5 b}$ \\
\hline$\alpha_{\mathrm{G}}$ & Chromatic adaptation parameter from red to green & Dimensionless & $.57^{\mathrm{b}}$ \\
\hline$\alpha_{\mathrm{R}}$ & Chromatic adaptation parameter from green to red & Dimensionless & $.30^{\mathrm{b}}$ \\
\hline
\end{tabular}

Note: PAR $=$ photosynthetically active radiation.

${ }^{a}$ Measured parameter.

b Estimated parameter (see "Methods").

c The iterative fitting procedure led to an estimate of $p_{\max }$ that diverged to infinity. This indicated that the light intensities encountered were still in the linear part of the $p_{i}(I)$ curve, such that $p_{\max }$ was not reached. Hence, $p_{\max }$ of the species was arbitrarily set to a high (but not unrealistic) value of $0.080 \mathrm{~h}^{-1}$ to guarantee that the modeled $p_{i}(I)$ values remained in the linear part.

pico mainly absorbs red light (fig. $1 B$ ), the model predicts that its exclusion rate as a function of $T$ will show the same pattern as the average red-light absorption by the flexible phenotype (cf. green lines in fig. 7B, 7C). Likewise, the exclusion rate of the red pico will show the same pattern as the average green light absorption by the flexible phenotype (cf. red lines in fig. $7 B, 7 C$ ). The competition experiments confirmed that competitive exclusion rates remained independent of the fluctuation period for $T<$ 14 days, while the competitive exclusion rate was higher at $T=28$ days. Also in line with the model predictions, the red pico was excluded faster than the green pico (fig. 7C).

What is the advantage of chromatic adaptation? The benefit of phenotypic plasticity can be quantified by comparing the performance of a flexible phenotype with a fixed phenotype that has lost its plasticity. Terauchi et al. (2004) discovered a photoreceptor involved in the complementary chromatic adaptation of cyanobacteria. Knockout mutants lacking the photoreceptor had lost their phenotypic plasticity and displayed a fixed intermediate pigment composition with equal amounts of phycoerythrin and phycocyanin. Accordingly, we calculated the competitive exclusion rates of the red and green picos in case our flexible phenotype would not be capable of chromatic adaptation but would have a fixed intermediate pigment composition (i.e., a fixed phycoerythrin fraction of 0.5 ; dashed lines in fig. $7 C$ ). This shows that when fluctuations are fast $(T<14$ days), chromatic adaptation does not affect the rate of competitive exclusion. Only when the period of the fluctuations exceeds $T>14$ days does chromatic adaptation make a difference (cf. solid lines and dashed lines in fig. 7C). Thus, chromatic adaptation is advantageous in competition only when there is sufficient time for the flexible phenotype to fully adjust its pigmentation to the prevailing light color. 


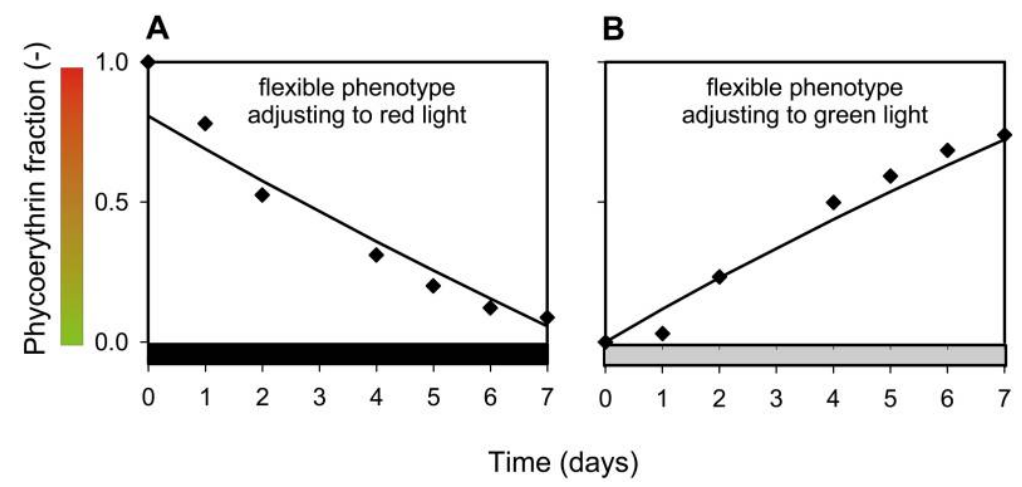

Figure 4: Chromatic adaptation of the flexible phenotype. $A$, Green-adapted monoculture exposed to red light. $B$, Red-adapted monoculture exposed to green light. Diamonds represent experimental data, and lines represent model predictions. Bars at the bottom of the panels indicate illumination by red light (black bar) and green light (gray bar). The pigment composition of the flexible phenotype is expressed by the phycoerythrin fraction $x$.

\section{Discussion}

\section{Timescale of Phenotypic Plasticity}

In this study, we have shown that the timescale of phenotypic plasticity affects competition in fluctuating environments. Theory often assumes a cost to phenotypic plasticity. Therefore, ideally one would like to perform experiments in which the fixed phenotype is a better competitor under constant conditions while the flexible phenotype is a better competitor in a variable environment. In practice, however, one has to work with the species one can find. Our experiments were based on cyanobacteria isolated from the same environment. In the Baltic Sea, we found a colorful mixture of red and green picocyanobac- teria with a constant pigment composition (fixed phenotypes) and filamentous cyanobacteria that can tune their pigments to the prevailing light color (flexible phenotypes). In our experiments, it turned out that the flexible phenotype was the strongest competitor under all experimental conditions. Yet, the experiments clearly revealed that the rate of competitive exclusion depended on the timescale of phenotypic plasticity in relation to the frequency of environmental fluctuations (fig. 7). When environmental fluctuations were fast compared with the rate of chromatic adaptation, the flexible phenotype displayed an intermediate pigment composition and, hence, could not benefit from its plasticity. In contrast, when environmental fluctuations were slow, the flexible phenotype had

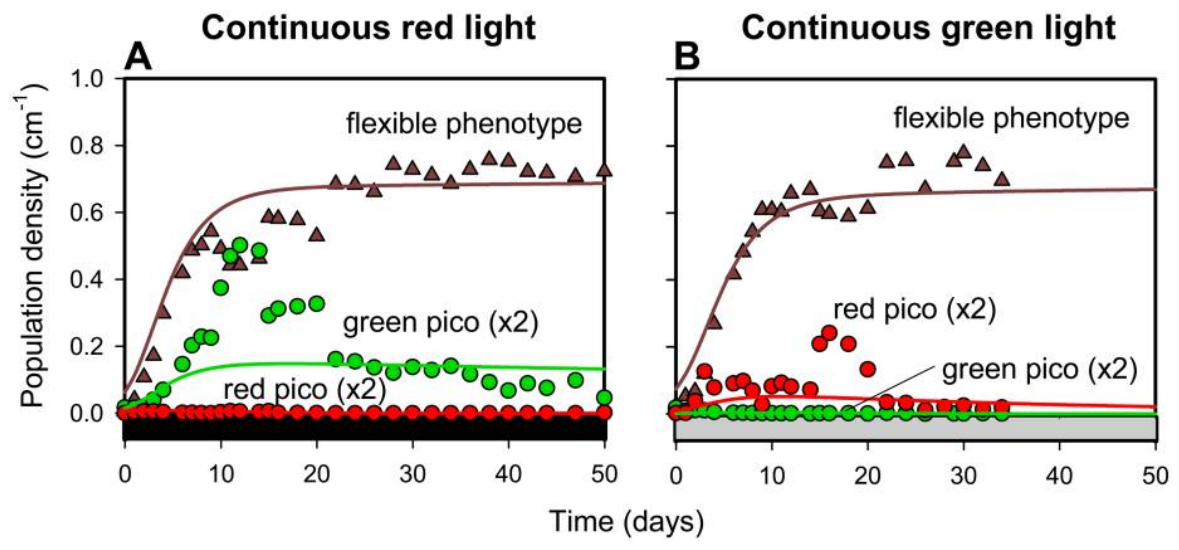

Figure 5: Competition experiments between the green pico (green circles and line), red pico (red circles and line), and flexible phenotype (brown triangles and line) exposed to continuous red light $(A)$ and continuous green light $(B)$. Symbols represent experimental data, while lines represent model predictions. Population densities are expressed by their light absorption. Bars at the bottom of the panels indicate illumination by red light (black bar) and green light (gray bar). 

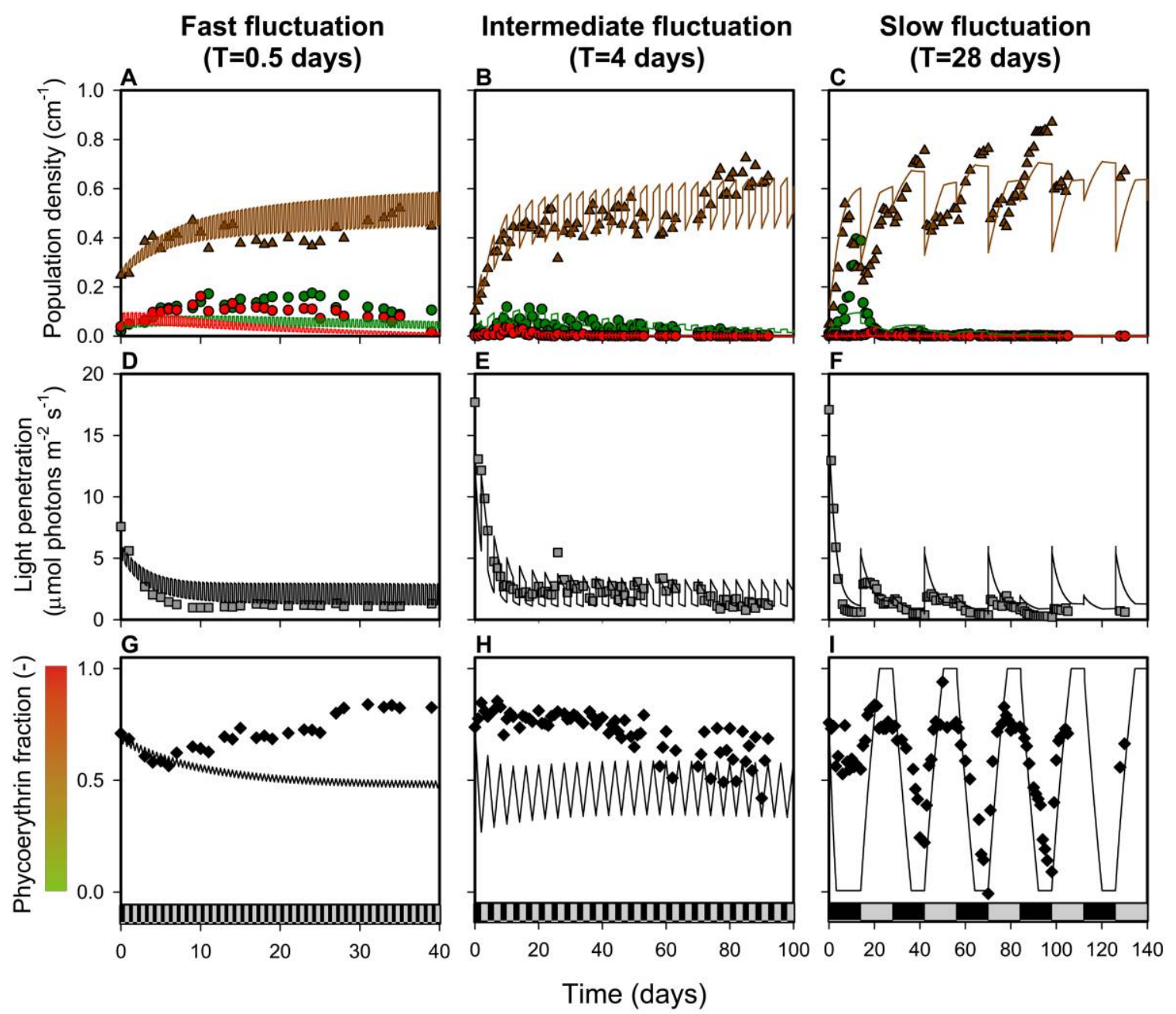

Figure 6: Competition experiments between the green pico, red pico, and flexible phenotype exposed to fluctuations in light color. The competition experiments were exposed to fast fluctuations (left panels), intermediate fluctuations (middle panels), and slow fluctuations (right panels). A-C, Population densities of the green pico (green circles), red pico (red circles), and flexible phenotype (brown triangles). Population densities are expressed by their light absorption. $D-F$, Light intensity penetrating through the culture vessel (gray squares). G-I, Pigment composition of the flexible phenotype (black diamonds) during the competition experiments. Lines represent the model predictions. Bars at the bottom of the panels indicate illumination by red light (black) and green light (gray).

sufficient time to fully adjust its pigmentation to the prevailing light color. In this case, the flexible phenotype benefited from its phenotypic plasticity, since it performed better than a fixed phenotype of intermediate pigment composition (cf. solid lines and dashed lines in fig. 7C).

Model studies of Padilla and Adolph (1996) and Gabriel $(2005,2006)$ compared the fitness of a phenotypically fixed individual with that of a flexible phenotype switching from one phenotypic state to the other state after a certain time lag. They showed that the advantage of phenotypic plasticity decreased with increasing environmental variability and also decreased with an increasing time lag in its phenotypic adjustment. In highly variable environments, plas- ticity could even be disadvantageous because of a permanent mismatch between the flexible but time-lagged phenotype and its environment.

Our results confirm these findings. Although the flexible phenotype was the strongest competitor, it benefited from its phenotypic plasticity only if environmental fluctuations were sufficiently slow compared with the rate of phenotypic adjustment. However, in contrast to the model studies of Padilla and Adolph (1996) and Gabriel $(2005,2006)$, rapid fluctuations in environmental conditions did not have a negative impact on the fitness of the flexible phenotype. In their model studies, the flexible phenotype switched abruptly from one phenotypic state to the other 

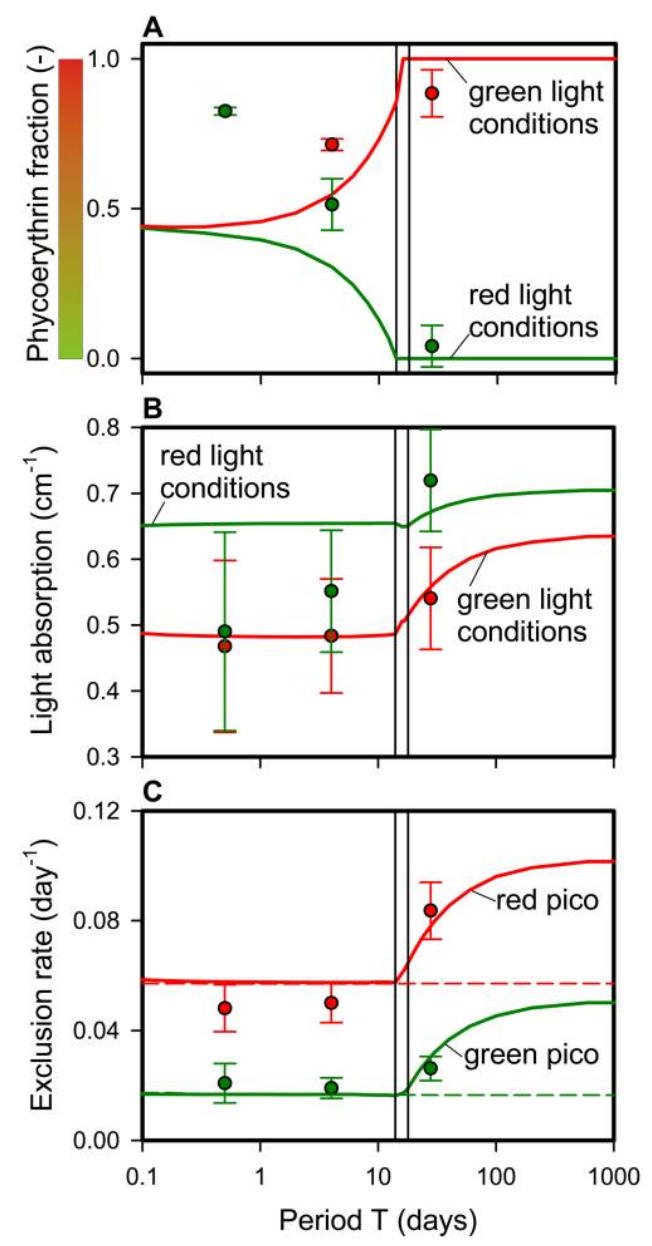

Figure 7: Implications of chromatic adaptation at different fluctuation frequencies. A, Amplitude of the fluctuations in pigment composition of the flexible phenotype, as expressed by the minimum values (green circles and lines) and maximum values (red circles and lines) of its phycoerythrin fraction. $B$, Time-averaged light absorption by the flexible phenotype during green light conditions (red circles and line) and red light conditions (green circles and line). $C$, Rates of competitive exclusion of the red pico (red circles and lines) and green pico (green circles and lines) in the competition experiments. Lines indicate model predictions, and symbols represent the experimental data $( \pm S D)$ from the competition experiments with fast, intermediate, and slow fluctuation regimes. The first vertical line (at $T=14$ days) indicates the periodicity at which the flexible phenotype becomes fully adapted to red light conditions, while the second vertical line (at $T=18$ days) indicates the periodicity at which it becomes fully adapted to green light conditions. The horizontal dashed lines in $C$ indicate the predicted rates of competitive exclusion in case the flexible phenotype would have been unable of chromatic adaptation (assuming a fixed phycoerythrin fraction of $x=0.5$ ).

state. In our system, the flexible phenotype gradually adjusted its pigment composition to the prevailing light conditions. As a result, the flexible phenotype displayed an intermediate pigment composition when fluctuations were too fast for full adjustment. This intermediate pigment composition prevented a complete mismatch between the flexible phenotype and its variable environment and thereby explains why rapid fluctuations never had a negative fitness impact in our study system.

When is reversible phenotypic plasticity advantageous during species interactions in fluctuating environments? Let us define the timescale of phenotypic adjustment as the time required to change from one phenotype to the other. According to our results, phenotypic plasticity is advantageous only if the period of the environmental fluctuations $\left(T_{\mathrm{f}}\right)$ exceeds twice the timescale of phenotypic adjustment $\left(T_{\mathrm{a}}\right)$ :

$$
\frac{T_{\mathrm{f}}}{2 T_{\mathrm{a}}} \gg 1
$$

In this case, there is sufficient time for the flexible phenotype to fully adjust its phenotype to both the ups and downs in the prevailing environmental conditions.

\section{The Ecological Significance of Chromatic Adaptation}

Complementary chromatic adaptation (CCA) of cyanobacteria is extensively studied at the molecular and physiological levels (Tandeau de Marsac 1977; Kehoe and Gutu 2006). Yet, surprisingly little is known about the ecology and biogeographical distribution of species capable of CCA. Can we predict, on the basis of the findings of this study, in which environments CCA will be advantageous?

In natural waters, phytoplankton species are exposed to fluctuating light conditions when cells change their vertical position in the water column. In the Baltic Sea, from which our Pseudanabaena strain and picocyanobacteria originated, the underwater light spectrum changes from white light at the surface to green light at greater depths (Stomp et al. 2007b; Haverkamp et al. 2008). At the water surface, in white light, we would expect that the flexible phenotype will adjust its pigment composition to complement the pigments of competing phytoplankton species, consistent with earlier competition experiments (Stomp et al. 2004). Accordingly, its pigment composition at the surface may depend on the pigment composition and population dynamics of its competitors. Deeper down in the water column, the prevailing green light conditions in the Baltic Sea perfectly match the absorption peak of the phycoerythrin pigment (Stomp et al. 2007a, 2007b). Hence, we expect that phenotypically flexible species like our Pseudanabaena strain will turn red (i.e., will produce the red pigment phycoerythrin) when mixed to greater depth in the Baltic Sea. This may also explain why Pseudanabaena was biased toward red pigmentation in our experiments 


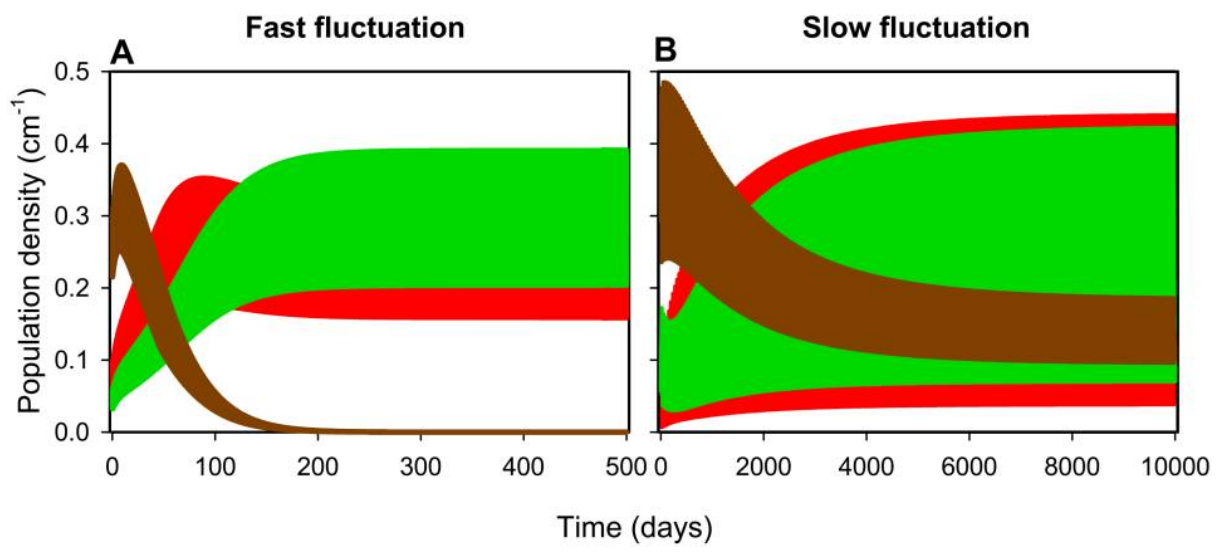

Figure 8: Model simulations exploring the possibility of coexistence in a fluctuating light environment. $A$, When fluctuations are fast $(T=0.5$ days), the model predicts coexistence of the red pico (red) and green pico (green), which jointly exclude the flexible phenotype (brown line). B, When fluctuations are slow ( $T=40$ days), the model predicts coexistence of all three species. In both panels, the species fluctuations are fast compared with the timescale of the $X$-axis, so that the species abundances are merged into bands. Population abundances are expressed in terms of the light absorption by each species. The model parameters in these simulations differed from the species parameters estimated in the monoculture experiments. In particular, these simulations assumed that the flexible phenotype was a weaker competitor for green light than the red pico and a weaker competitor for red light than the green pico. Model parameters are the same as in table 2, except for the photosynthetic efficiency of the

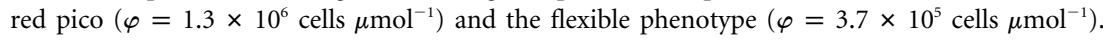

(figs. 6, 7), because it can encounter green light but not purely red light in its natural habitat.

Although green light is the predominant underwater light color in coastal seas like the Baltic Sea, other light colors may prevail in other aquatic ecosystems. For instance, blue light is the predominant color in the open ocean, whereas red light penetrates the deepest in peat lakes (Stomp et al. 2007a, 2007b). These different underwater light environments are likely to select for chromatic adaptation by specific sets of pigments. For example, in clear ocean waters, it would be advantageous for cyanobacteria to tune pigments active in the blue part of their light absorption spectrum. Indeed, some marine Synechococcus strains from oceanic environments are flexible in their absorption of blue versus green light. They use two alternative chromophores in the phycoerythrin pigments (Palenik 2001). The chromophore phycoerythrobilin (PEB) has its absorption peak in green light $(550 \mathrm{~nm})$, while the chromophore phycourobilin (PUB) has its absorption peak shifted toward blue light $(495 \mathrm{~nm})$. These flexible phenotypes have a low PUB/PEB ratio when exposed to white light at the water surface but a high PUB/ PEB ratio when exposed to blue light at greater depths (Palenik 2001; Everroad et al. 2006).

What is the ecological significance of the timescale of chromatic adaptation? Some cyanobacteria are neutrally buoyant. They are passively dispersed throughout the euphotic zone by turbulent mixing. The timescale of turbulent mixing may vary from several hours to several days
(Denman and Gargett 1983; Huisman et al. 1999b), depending on the intensity of turbulent mixing and the depth of the euphotic zone. When mixing through the euphotic zone occurs on a timescale of hours, cells will experience fluctuations in the underwater light color that will be too fast for CCA. In contrast, when mixing through the euphotic zone occurs on a timescale of several days, cells will experience slower fluctuations in the underwater light color that can be tracked by CCA. The latter condition may apply to stratified open ocean waters, where turbulent mixing can be relatively weak and the euphotic zone may extend over more than $100 \mathrm{~m}$ depth. Here, CCA by marine Synechococcus species with flexible PUB/PEB ratios could be advantageous compared to Synechococcus species with a fixed pigment composition (Everroad et al. 2006).

Complementary chromatic adaptation may be particularly advantageous for positively buoyant cyanobacteria that can escape from turbulent mixing. For instance, cyanobacteria with gas vesicles may float upwards, forming blooms near the water surface as long as the weather conditions remain stable (Huisman et al. 2004; Jöhnk et al. 2008). When these surface blooms are disrupted by storm events, their light environment may change completely as they are entrained by intense mixing to the deeper waters below. Storm events occur irregularly, at intervals of, say, several weeks or months. Our results indicate that, at these timescales, CCA would provide a strong selective advantage. We therefore hypothesize that this scenario of intermittent mixing, which seems to apply to the Baltic Sea 
(Walsby et al. 1997), will favor chromatic adaptation in buoyant cyanobacteria such as our Pseudanabaena strain.

\section{Coexistence versus Competitive Exclusion}

Temporal variability can promote species coexistence, depending on the frequency and amplitude of the environmental fluctuations (Hutchinson 1961; Connell 1978; Armstrong and McGehee 1980). This is confirmed by many experimental studies that have shown that a fluctuating resource supply yields a higher species diversity in phytoplankton communities than a constant resource supply (Sommer 1984; Gaedeke and Sommer 1986; Grover 1991; Litchman 1998, 2003; Flöder et al. 2002). Likewise, theoretical studies predict that competition for two fluctuating resources may allow coexistence of a generalist and two specialist species (Wilson and Yoshimura 1994; Egas et al. 2004; Abrams 2006a, 2006b). These theoretical studies may resemble our study, in which the flexible phenotype can be interpreted as a generalist species capable of growing on both resources and the red pico and green pico can be considered specialists growing mainly on green light and on red light, respectively.

However, in our competition experiments and parameterized model simulations, we did not find coexistence under fluctuating conditions. An explanation for the lack of coexistence could be the absence of suitable trade-offs. Model studies have shown that coexistence in fluctuating environments strongly depends on the parameter combinations of competing species (Grover 1990; Litchman and Klausmeier 2001). In particular, a strong trade-off is required such that each species has an advantage during at least part of the competitive process. In our experiments, analogous to the $\mathrm{R}^{*}$ concept of competition for nutrients (Tilman 1982), the species with the lowest critical light intensity for a particular color of light will be the superior competitor for that color of light (Huisman and Weissing 1994). In view of the critical light intensities (table 1), the flexible phenotype and the green pico were equally strong competitors for red light, as confirmed by their neutral coexistence in continuous red light (fig. 5A). In addition, the flexible phenotype was a slightly stronger competitor for green light than the red pico (fig. $5 B$ ). This makes the flexible phenotype a superior competitor under fluctuating light conditions. As a result, in our experiments, fluctuating light conditions did not promote coexistence; the flexible phenotype always won.

Hypothetically, we may expect many situations in which there will be trade-offs in the competitive ability for different colors of light. In particular, it seems plausible that flexible phenotypes will often be weaker competitors for a single color of light than species specialized on that light color. We simulated such a situation in which we used our estimated model parameters but modified the photosynthetic efficiencies of the species to mimic this trade-off. With this new parameter setting, the flexible phenotype was a weaker competitor for red light than the green pico and a weaker competitor for green light than the red pico. In this case, with fast fluctuations of red and green light, the model predicts that the flexible phenotype will not be able to catch up with the changing light conditions and is excluded by the joint forces of the coexisting red and green picos (fig. 8A). However, at slower fluctuations of red and green light, the model predicted coexistence of all three species (fig. $8 \mathrm{~B}$ ). Additional model simulations (not shown) revealed that the transition from competitive exclusion of the flexible phenotype to coexistence of all three species occurred at a periodicity of $\sim 14$ days, the same periodicity at which the flexible phenotype starts to benefit from chromatic adaptation in our experiments (fig. 7). These model results support the earlier model prediction that two specialists and one generalist can coexist on two fluctuating resources (Wilson and Yoshimura 1994; Egas et al. 2004; Abrams 2006a, 2006b). Moreover, coexistence of all three species requires that the flexible phenotype has sufficient time to fully adapt to the prevailing light colors.

\section{Conclusions}

In conclusion, our results confirm that the underwater light color is an important selective factor in phytoplankton competition. Phytoplankton species that have tuned their photosynthetic pigments to the prevailing light color have a clear competitive advantage. More generally, our results show that the timescale of phenotypic plasticity can be decisive for species interactions in fluctuating environments. In particular, the interplay between the timescale of phenotypic plasticity and the timescale of environmental fluctuations influences the rate of competitive exclusion, may reverse competitive hierarchies, and can affect the species composition of ecological communities.

\section{Acknowledgments}

We thank L. J. Stal for providing the three cyanobacterial strains used in our experiments, C. Klausmeier and E. Litchman for discussion, P. Stol for his help with a series of pilot experiments, and students of the MSc course in Limnology and Oceanography 2005 for their help with measurements of the adaptation rate of Pseudanabaena. We thank A. M. De Roos, L. Jiang, and an anonymous reviewer for their helpful comments on the manuscript. The research of M.S. and J.H. was supported by the Earth and Life Sciences Foundation (ALW), which is subsidized by the Netherlands Organization for Scientific Research (NWO). 


\section{APPENDIX}

\section{Chemostat Conditions}

We used a nutrient-rich mineral medium to avoid nutrient limitation (Stomp et al. 2004). We added $\mathrm{NaCl}$ to obtain a salinity of $\sim 12 \mathrm{~g} \mathrm{~L}^{-1}$, which resembles the brackish waters of the Baltic Sea. The chemostat vessels were bubbled with compressed air enriched with $\mathrm{CO}_{2}$ to avoid carbon limitation. The $\mathrm{CO}_{2}$ inflow was controlled by mass flow controllers (BROOKS Smart Mass Flow) maintaining the $\mathrm{pH}$ between 8 and 8.5. The chemostat vessels were maintained at a constant temperature of $21^{\circ} \mathrm{C}$ by water jackets placed between the light sources and the chemostat vessel and connected to a Colora thermocryostat.

\section{Background Turbidity}

The absorption spectrum of the background turbidity, $K_{\mathrm{bg}}(\lambda)$, was calculated from the spectrum of the incident light intensity, $I_{\text {in }}(\lambda)$, and the spectrum of light penetrating through the chemostat vessels, $I_{\text {out }}(\lambda)$, when filled with mineral medium only:

$$
K_{\mathrm{bg}}(\lambda)=\frac{\ln \left(I_{\mathrm{in}}(\lambda)\right)-\ln \left(I_{\mathrm{out}}(\lambda)\right)}{z_{\mathrm{m}}} .
$$

The background absorption in our shallow chemostat vessels was largely independent of wavelength and thus could be treated as a wavelength-independent parameter $K_{\mathrm{bg}}$.

\section{Population Densities}

Samples were taken from the chemostat experiments daily and were fixed with a solution of $10 \%$ gluteraldehyde and 1\% formaldehyde (Tsuji and Yanagita 1981). The population densities of the species were counted in duplicate with a MoFlow flowcytometer (Dako Cytomation, Fort Collins, CO) equipped with a blue laser $(488 \mathrm{~nm})$ and a red laser $(633 \mathrm{~nm})$. The flow cytometer could distinguish between red and green cyanobacteria on the basis of their different fluorescence signals (Jonker et al. 1995; Stomp et al. 2004). Cells rich in phycoerythrin emitted yelloworange light (550-620 nm) when excited by the blue laser, whereas cells rich in phycocyanin emitted far-red light (>670 nm) when excited by the red laser. The flow cytometer distinguished between the single-celled picocyanobacteria and the much larger filaments of Pseudanabaena by their size (using side scattering). Since the picocyanobacteria were much smaller than the Pseudanabaena filaments, the counts obtained by flow cytometry were not a good indicator of the biomasses of the three species. For comparison, therefore, the population densities of the species were converted to the total light ab- sorption (per $\mathrm{cm}$ ) by each species, $A_{i}$, which can be expressed as

$$
A_{i}=\frac{1}{z_{\mathrm{m}}} \ln \left(\frac{\int_{400}^{700} I_{\mathrm{in}}(\lambda) d \lambda}{\int_{400}^{700} I_{\mathrm{in}}(\lambda) e^{-k_{i}(\lambda) N_{i} z_{\mathrm{m}}} d \lambda}\right)
$$

The fluorescence signals of the Pseudanabaena filaments obtained from the blue and red lasers provided information on the relative content of phycoerythrin and phycocyanin in Pseudanabaena. Using monoculture experiments of Pseudanabaena grown under different red light and green light conditions, we calibrated these fluorescence signals with absorption spectra measured with the AMINCO DW-2000 double-beam spectrophotometer. This enabled estimation of the fraction phycoerythrin (i.e., the parameter $x_{i}$ in eq. [4]), and hence the absorption spectrum of Pseudanabaena during competition could be reconstructed from the flow-cytometer measurements.

\section{Literature Cited}

Abrams, P. A. 1999. The adaptive dynamics of consumer choice. American Naturalist 153:83-97.

- 2006a. Adaptive change in the resource-exploitation traits of a generalist consumer: the evolution and coexistence of generalists and specialists. Evolution 60:427-439.

. 2006b. The prerequisites for and likelihood of generalistspecialist coexistence. American Naturalist 167:329-342.

Acinas, S. G., T. H. A. Haverkamp, J. Huisman, and L. J. Stal. 2008. Phenotypic and genetic diversification of Pseudanabaena spp. (cyanobacteria). ISME Journal, doi:10.1038/ismej.2008.78.

Agawin, N. S. R., S. Rabouille, M. J. W. Veldhuis, L. Servatius, S. Hol, H. M. J. van Overzee, and J. Huisman. 2007. Competition and facilitation between unicellular nitrogen-fixing cyanobacteria and non-nitrogen-fixing phytoplankton species. Limnology and Oceanography 52:2233-2248.

Agrawal, A. A. 2001. Phenotypic plasticity in the interactions and evolution of species. Science 294:321-326.

Armstrong, R. A., and R. McGehee. 1980. Competitive exclusion. American Naturalist 115:151-170.

Béjà, O., E. N. Spudich, J. L. Spudich, M. Leclerc, and E. F. DeLong. 2001. Proteorhodopsin phototrophy in the ocean. Nature 411:786789.

Chesson, P., R. L. E. Gebauer, S. Schwinning, N. Huntly, K. Wiegand, M. S. K. Ernest, A. Sher, A. Novoplansky, and J. F. Weltzin. 2004. Resource pulses, species interactions, and diversity maintenance in arid and semi-arid environments. Oecologia (Berlin) 141:236253.

Connell, J. H. 1978. Diversity in tropical rain forests and coral reefs. Science 199:1302-1310.

Denman, K. L., and A. E. Gargett. 1983. Time and space scales of vertical mixing and advection of phytoplankton in the upper ocean. Limnology and Oceanography 28:801-815.

Dring, M. J. 1981. Chromatic adaptation of photosynthesis in benthic marine algae: an examination of its ecological significance using a theoretical model. Limnology and Oceanography 26:271-284.

Egas, M., U. Dieckmann, and M. W. Sabelis. 2004. Evolution restricts 
the coexistence of specialists and generalists: the role of trade-off structure. American Naturalist 163:518-531.

Egas, M., M. W. Sabelis, and U. Dieckmann. 2005. Evolution of specialization and ecological character displacement of herbivores along a gradient of plant quality. Evolution 59:507-520.

Ernst, A., S. Becker, U. I. A. Wollenzien, and C. Postius. 2003. Ecosystem-dependent adaptive radiations of picocyanobacteria inferred from 16S rRNA and ITS-1 sequence analysis. Microbiology 149:217-228.

Everroad, C., C. Six, F. Partensky, J. C. Thomas, J. Holtzendorff, and A. M. Wood. 2006. Biochemical bases of Type IV chromatic adaptation in marine Synechococcus spp. Journal of Bacteriology 188: 3345-3356.

Flöder, S., J. Urabe, and Z. Kawabata. 2002. The influence of fluctuating light intensities on species composition and diversity of natural phytoplankton communities. Oecologia (Berlin) 133:395401.

Gabriel, W. 2005. How stress selects for reversible phenotypic plasticity. Journal of Evolutionary Biology 18:873-883.

-2006. Selective advantage of irreversible and reversible phenotypic plasticity. Archiv fuer Hydrobiologie 167:1-20.

Gaedeke, A., and U. Sommer. 1986. The influence of the frequency of periodic disturbances on the maintenance of phytoplankton diversity. Oecologia (Berlin) 71:25-28.

Gaiducov, N. 1902. Uber den Einfluss farbigen Lichtes auf die Farbung lebender Oscillarien. Abhandlungen der Königlich-Preussischen Akademie der Wissenschaften 5:1-36.

Gélinas, M., B. Pinel-Alloul, and M. Slusarczyk. 2007. Alternative antipredator responses of two coexisting Daphnia species to negative size selection by YOY perch. Journal of Plankton Research 29:775-789.

Grossman, A. R., M. R. Schaeffer, G. G. Chiang, and J. L. Collier. 1993. The phycobilisome, a light-harvesting complex responsive to environmental conditions. Microbiological Reviews 57:725-749.

Grover, J. P. 1988. Dynamics of competition in a variable environment: experiments with two diatom species. Ecology 69:408-417.

1990. Resource competition in a variable environment: phytoplankton growing according to Monod's model. American Naturalist 136:771-789.

1991. Dynamics of competition among microalgae in variable environments: experimental tests of alternative models. Oikos 62:231-243.

Haverkamp, T., S. G. Acinas, M. Doeleman, M. Stomp, J. Huisman, and L. J. Stal. 2008. Diversity and phylogeny of Baltic Sea picocyanobacteria inferred from their ITS and phycobiliprotein operons. Environmental Microbiology 10:174-188.

Hessen, D. O., and E. Van Donk. 1993. Morphological changes in Scenedesmus induced by substances released from Daphnia. Archiv fuer Hydrobiologie 127:129-140.

Huisman, J., and F. J. Weissing. 1994. Light-limited growth and competition for light in well-mixed aquatic environments: an elementary model. Ecology 75:507-520.

- 1995. Competition for nutrients and light in a mixed water column: a theoretical analysis. American Naturalist 146:536-564.

Huisman, J., R. R. Jonker, C. Zonneveld, and F. J. Weissing. 1999a. Competition for light between phytoplankton species: experimental tests of mechanistic theory. Ecology 80:211-222.

Huisman, J., P. van Oostveen, and F. J. Weissing. 1999b. Critical depth and critical turbulence: two different mechanisms for the devel- opment of phytoplankton blooms. Limnology and Oceanography 44:1781-1787.

Huisman, J., J. Sharples, J. M. Stroom, P. M. Visser, W. E. A. Kardinaal, J. M. H. Verspagen, and B. Sommeijer. 2004. Changes in turbulent mixing shift competition for light between phytoplankton species. Ecology 85:2960-2970.

Hutchinson, G. E. 1961. The paradox of the plankton. American Naturalist 95:137-145.

Jöhnk, K. D., J. Huisman, J. Sharples, B. Sommeijer, P. M. Visser, and J. M. Stroom. 2008. Summer heatwaves promote blooms of harmful cyanobacteria. Global Change Biology 14:495-512.

Jonker, R. R., J. T. Meulemans, G. B. J. Dubelaar, M. F. Wilkins, and J. Ringelberg. 1995. Flow cytometry: a powerful tool in analysis of biomass distributions in phytoplankton. Water Science and Technology 32:177-182.

Kehoe, D. M., and A. Gutu. 2006. Responding to color: the regulation of complementary chromatic adaptation. Annual Review of Plant Biology 57:127-150.

Kirk, J. T. O. 1994. Light and photosynthesis in aquatic ecosystems. 2nd ed. Cambridge University Press, Cambridge.

Laforsch, C., and R. Tollrian. 2004. Inducible defenses in multipredator environments: cyclomorphosis in Daphnia cucullata. Ecology 85:2302-2311.

Lampert, W., K. O. Rothhaupt, and E. von Elert. 1994. Chemical induction of colony formation in a green alga (Scenedesmus acutus) by grazers (Daphnia). Limnology and Oceanography 39:15431550.

Litchman, E. 1998. Population and community responses of phytoplankton to fluctuating light. Oecologia (Berlin) 117:247-257. 2003. Competition and coexistence of phytoplankton under fluctuating light: experiments with two cyanobacteria. Aquatic Microbial Ecology 31:241-248.

Litchman, E., and C. A. Klausmeier. 2001. Competition of phytoplankton under fluctuating light. American Naturalist 157:170187.

Metz, J. A. J., R. M. Nisbet, and S. A. H. Geritz. 1992. How should we define fitness for general ecological scenarios? Trends in Ecology \& Evolution 7:198-202.

Miner, B. G., S. E. Sultan, S. G. Morgan, D. K. Padilla, and R. A. Relyea. 2005. Ecological consequences of phenotypic plasticity. Trends in Ecology \& Evolution 20:685-692.

Montesinos, E., R. Guerrero, C. Abella, and I. Esteve. 1983. Ecology and physiology of the competition for light between Chlorobium limicola and Chlorobium phaeobacteroides in natural habitats. Applied and Environmental Microbiology 46:1007-1016.

Padilla, D. K., and S. C. Adolph. 1996. Plastic inducible morphologies are not always adaptive: the importance of time delays in a stochastic environment. Evolutionary Ecology 10:105-117.

Palenik, B. 2001. Chromatic adaptation in marine Synechococcus strains. Applied and Environmental Microbiology 67:991-994.

Passarge, J., S. Hol, M. Escher, and J. Huisman. 2006. Competition for nutrients and light: stable coexistence, alternative stable states, or competitive exclusion? Ecological Monographs 76:57-72.

Pick, F. R. 1991. The abundance and composition of freshwater picocyanobacteria in relation to light penetration. Limnology and Oceanography 36:1457-1462.

Ramus, J. 1983. A physiological test of the theory of complementary chromatic adaptation. II. Brown, green and red seaweeds. Journal of Phycology 19:173-178.

Rocap, G., F. W. Larimer, J. Lamerdin, S. Malfatti, P. Chain, N. A. 
Ahlgren, A. Arellano, et al. 2003. Genome divergence in two Prochlorococcus ecotypes reflects oceanic niche differentiation. Nature 424:1042-1047.

Sathyendranath, S., and T. Platt. 1989. Computation of aquatic primary production: extended formalism to include effect of angular and spectral distribution of light. Limnology and Oceanography 34:188-198.

Sommer, U. 1984. The paradox of the plankton: fluctuations of phosphorus availability maintain diversity in flow-through cultures. Limnology and Oceanography 29:633-636.

Stomp, M., J. Huisman, F. de Jongh, A. J. Veraart, D. Gerla, M. Rijkeboer, B. W. Ibelings, U. I. A. Wollenzien, and L. J. Stal. 2004. Adaptive divergence in pigment composition promotes phytoplankton biodiversity. Nature 432:104-107.

Stomp, M., J. Huisman, L. J. Stal, and H. C. P. Matthijs. $2007 a$. Colorful niches of phototrophic microorganisms shaped by vibrations of the water molecule. ISME Journal 1:271-282.

Stomp, M., J. Huisman, L. Vörös, F. R. Pick, M. Laamanen, T. Haverkamp, and L. J. Stal. 2007b. Colourful coexistence of red and green picocyanobacteria in lakes and seas. Ecology Letters 10:290298.

Sultan, S. E., and F. A. Bazzaz. 1993. Phenotypic plasticity in Polygonum persicaria. II. Norms of reaction to soil moisture and the maintenance of genetic diversity. Evolution 47:1032-1049.

Tandeau de Marsac, N. 1977. Occurrence and nature of chromatic adaptation in cyanobacteria. Journal of Bacteriology 130:82-91.

Terauchi, K., B. L. Montgomery, A. R. Grossman, J. C. Lagarias, and D. M. Kehoe. 2004. RcaE is a complementary chromatic adaptation photoreceptor required for green and red light responsiveness. Molecular Microbiology 51:567-577.
Tilman, D. 1982. Resource competition and community structure. Princeton University Press, Princeton, NJ.

Tsuji, T., and T. Yanagita. 1981. Improved fluorescent microscopy for measuring the standing stock of phytoplankton including fragile components. Marine Biology 64:207-211.

Van der Stap, I., M. Vos, and W. M. Mooij. 2007. Inducible defenses and rotifer food chain dynamics. Hydrobiologia 593:103-110.

Vila, X., and C. A. Abella. 2001. Light-harvesting adaptations of planktonic phototrophic micro-organisms to different light quality conditions. Hydrobiologia 452:15-30.

Vörös, L., C. Callieri, K. V. Balogh, and R. Bertoni. 1998. Freshwater picocyanobacteria along a trophic gradient and light quality range. Hydrobiologia 370:117-125.

Walsby, A. E., P. K. Hayes, R. Boje, and L. J. Stal. 1997. The selective advantage of buoyancy provided by gas vesicles for planktonic cyanobacteria in the Baltic Sea. New Phytologist 136:407-417.

Wilson, S. D., and J. Yoshimura. 1994. On the coexistence of specialists and generalists. American Naturalist 144:692-707.

Woltereck, R. 1909. Weitere experimentelle Untersuchungen über Artveränderung, speziell über das Wesen quantitativer Artunterschiede bei Daphnien. Verhandlungen der Deutschen Zoologischen Gesellschaft 19:110-173.

Wood, A. M., D. A. Phinney, and C. S. Yentsch. 1998. Water column transparency and the distribution of spectrally distinct forms of phycoerythrin-containing organisms. Marine Ecology Progress Series 162:25-31. 